\title{
Total ankle replacement - surgical treatment and rehabilitation
}

\author{
Agnieszka Prusinowska, Zbigniew Krogulec, Piotr Turski, Emil Przepiórski, Paweł Małdyk, \\ Krystyna Księżopolska-Orłowska
}

Department of Rheumatological Rehabilitation, Department and Polyclinic of Orthopaedic Rheumatology, Institute of Rheumatology in Warsaw, Poland

\begin{abstract}
Functions of the ankle joint are closely connected with the gait and ability to maintain an upright position. Degenerative lesions of the joint directly contribute to postural disorders and greatly restrict propulsion of the foot, thus leading to abnormal gait.

Development of total ankle replacement is connected with the use of the method as an efficient treatment of joint injuries and continuation of achievements in hip and knee surgery. The total ankle replacement technique was introduced as an alternative to arthrodesis, i.e. surgical fixation, which made it possible to preserve joint mobility and to improve gait. Total ankle replacement is indicated in post-traumatic degenerative joint disease and joint destruction secondary to rheumatoid arthritis. In this paper, total ankle replacement and various types of currently used endoprostheses are discussed. The authors also describe principles of early postoperative rehabilitation as well as rehabilitation in the outpatient setting.
\end{abstract}

Key words: ankle replacement, surgical treatment, rehabilitation.

\section{Introduction}

The ankle joint is a synovial hinge joint with only one degree of freedom allowed. The joint has closely fitting articular surfaces because it is subject to large loads at the single support stage of the gait cycle. It not only supports the whole body weight but is also affected by forces resulting from kinetic energy dissipation upon sudden contact with the ground while walking, running or jumping [1, 2].

The talus joint is a perfect example of co-operation between the ligament system, muscles and the bony architecture of the joint ends. In a neutral position or dorsiflexion of the foot, the wider dimension of the trochlea tali is ideally gripped by the fork-like structure of tibial and fibular bony ends. It should be noted that the forklike structure - formed by the medial and lateral malleoli - shows some elasticity because the tibia and fibula are not connate but held in place by a syndesmosis [2].

The range of dorsal-plantar flexion motions depends mainly on the arc length of articular surfaces. The artic- ular surface of the tibia is a circle sector with the central angle of 70 degrees, with the cusp located on the axis of rotation, while the articular surface of the trochlea tali is a sector with a central angle of 140-150 degrees. By simple subtraction it can be concluded that the total range of dorsal-plantar flexion should be 70-80 degrees. Since the trochlea tali is more prominent in the posterior than in the anterior part, the range of extension is greater as compared to the flexion range [2].

Except for dorsal and plantar flexions of the ankle joint, the foot moves around the longitudinal axis of the tibia (adduction and abduction), and around its own axis (pronation and supination). Actually, a movement in one plane must be coupled with displacement in the other two. Therefore, adduction must be accompanied by supination and slight dorsiflexion. Execution of the combined three movements puts the foot in an inverted position (inversion) while abduction combined with pronation and plantar flexion puts the foot in an everted position (eversion) [2]. 


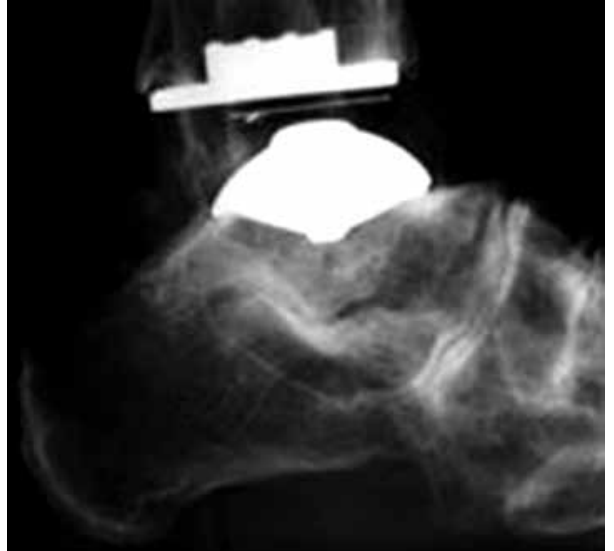

Fig. 1. STAR total ankle replacement $\left(2^{\text {nd }}\right.$ generation). Radiogram - lateral view.

Flexors of the talar joint are 4 times stronger than extensors because they must counteract the force of gravity, ligament pulling, and also due to shifting of movement from the talus, tarsal and crus bones by the structure of articular surfaces [3].

The talus bone is subject to major compressive forces, and plays a significant role in biomechanics of the foot. No muscles are attached, and the talus bone is surrounded by ligaments on their way to the distal part of the foot; therefore, it is called a "trapped bone", i.e. bound by ligaments. There are 13 muscles to which the mentioned ligaments belong. The talus bone is completely covered with articular surfaces and ligament insertion sites (ligamentous relay station). Due to the absence of muscle attachments, the talus bone is supplied by several direct arteries, through vessels entering it at ligament insertion sites. Such blood supply is sufficient in normal conditions. Fractures accompanied by additional subluxation may lead to blood flow impairment, and development of pseudarthrosis, or avascular necrosis of the talus bone [2].

\section{Total ankle replacement}

The first-generation ankle joint endoprostheses were developed in the 1970s. The endoprostheses consisted of metal tibial and talar elements with a polyethylene insert between them. The endoprostheses were characterised by excessive constraint, and they did not take account of the great rotational forces acting on the ankle joint. The possibility of making multi-axial movements compromised the endoprosthesis stability. It increased the incidence of implant loosening [4]. Despite poor outcomes of the use of the first-generation ankle joint endoprostheses, the interest in joint replacement did not decline, and led to development of second-generation total implants with an improved design and better methods

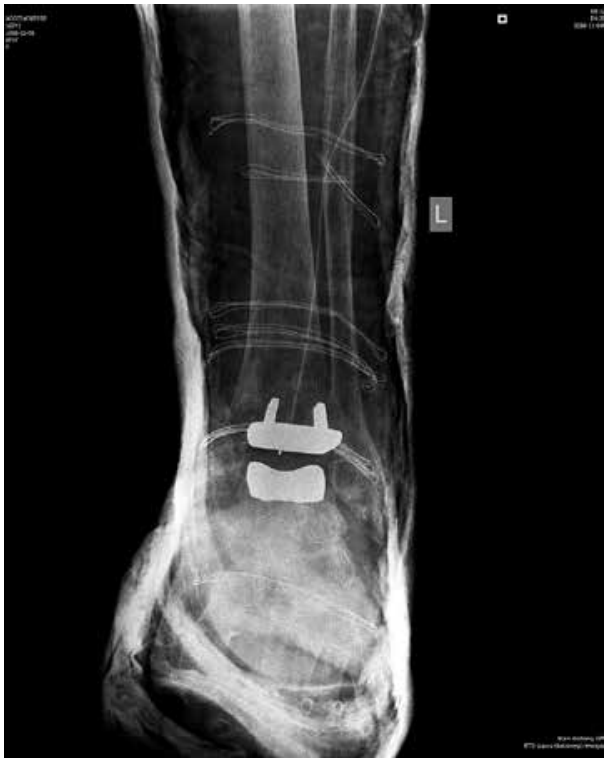

Fig. 2. TARIC total ankle replacement $\left(2^{\text {nd }}\right.$ generation). Radiogram - AP view.

for osseous integration (Figs. 1 and 2). More restrictive indications and contraindications for the surgery revived optimism about possible better outcomes [5].

In some of the $2^{\text {nd }}$-generation implants, mobile bearings were used between the tibial and talus elements, usually made of ultra-high molecular weight polyethylene (UHMWP), which increases stability of the joint, and theoretically contributes to reduced wear of the bearing [4]. A serious design fault of the model was the risk of subluxation and increased release of polyethylene particles. A mobile bearing causes increased wear of the rear part but can form highly congruent configurations, which considerably reduces the pressure surfaces [4]. A new type of bearing used in $2^{\text {nd }}$-generation endoprostheses is a fixed bearing which reduces the risk of breaking or luxation. Due to their exact congruence, the endoprostheses show a higher degree of osseous integration under strain, and reduced wear of the rear part of the bearing if there is an effective locking mechanism.

Fixed bearing implants have a single plane of motion between the polyethylene bearing inserted in the tibial component and the talus component [5]. With the mobile bearing type, there is a possibility of the bearing moving between the prosthesis elements, which increases the design complexity and poses a risk of endoprosthesis subluxation (Fig. 3).

Modern ankle prostheses consist of three elements: a polyethylene insert (mobile or fixed), a tibial component, and a talar component. Physiology of the joint mobility is characterised by the presence of rotational sliding and continuous change of axes of movement. Materials with osseointegrating properties have been 


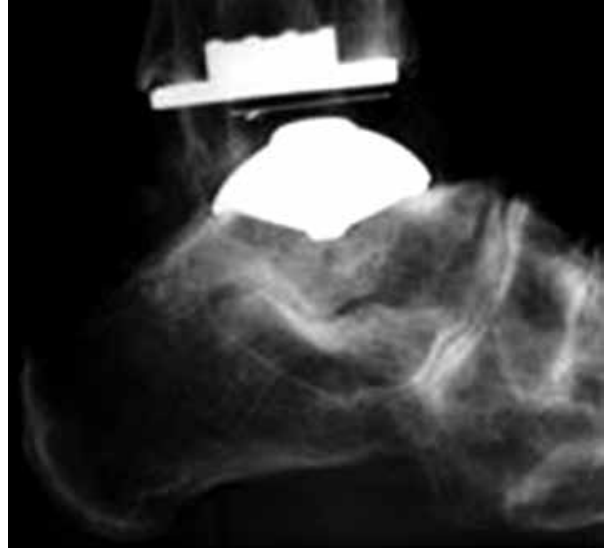

Fig. 3. TARIC total ankle replacement. Radiogram - lateral view.

applied to eliminate the use of cement, often coated with hydroxyapatite as a substance promoting bone ingrowth into implants.

Biomechanical factors taken into account when designing endoprostheses include congruence, that is, mutual adjustment, and adherence (constraint) of articular surfaces. Mutual adjustment of articular surfaces increases their contact area, and reduces the level of wear of the polyethylene insert. An ideal implant should have a congruent surface to reduce abrasion of the insert, and little tension of tissues around the ankle joint, which reduces the risk of component loosening.

Problems with development of an ankle joint prosthesis that mimics the physiological movement of the joint result from various factors connected with the joint anatomy. The ankle joint is subject to large loads on a limited area, which causes major mechanical loading of the prosthesis components, resulting in possible loosening of the implant. Due to its spongy structure, the proximal end of the tibia is not an ideal site for stable attachment of the prosthesis, while the fibula is poorly supplied with blood, especially in its medial part, and may pose a risk of impaired bone ingrowth into the talar component of the implant. Soft tissues surrounding the ankle joint are thin and prone to scarring, which, accompanied by wound healing impairment, may lead to stiffness of the joint.

Total ankle replacement is indicated in elderly patients (over 60 years of age), not very active physically, with adequate bone stock, without osteoporosis symptoms, with good blood supply, and correct axis of the operated joint. The patient should be slim (low body mass index - BMI) $[4,5]$. Deformities of the joint axis of the valgus or varus type of more than 15 degrees are difficult to correct. Apart from the above-mentioned situations, contraindications for the surgery include ischaemic lesions to the tibial or talus bone, avascular necrosis of the talus bone, neuropathies, Charcot joint, dysfunction of muscles of the lower extremity and the foot, major deformities in the frontal or axial plane, soft tissue losses, vascular failure of the extremity, active infection, and poor quality of soft tissues covering the ankle joint. Relative contraindications include osteoporosis, major instability of the ankle joint, history of ankle infection, poor skin condition, and intensive physical activity or heavy physical work [4, 5].

The age-related limitation seems controversial; however, the risk of complications is higher in younger patients due to their greater physical activity. Like any other surgical treatment, total ankle replacement may lead to complications. Typical complications include wound healing disorders, superficial and deep infections, aseptic loosening, subsidence of prosthetic components, and osteolysis of the bone tissue. There can also occur nerve injuries, and lateral or medial malleolar fractures. A major problem after total ankle replacement is decline of the talus bone resulting from inadequate balance of the soft tissue. It is a serious problem even to very experienced surgeons [5-7]. It should be stressed that loosening of ankle prosthetic elements occurs more often than in the case of implants used for hip or knee replacement. Total ankle replacement is a surgical procedure that requires technical skills, and the initial complication rate can be high. The incidence of intraoperative complications decreases along with increasing surgeon's experience. Then, positioning of the prosthetic component is performed better, and reproducibility of the procedure increases.

The first generation of ankle endoprostheses showed a high failure rate, and the rate of revision surgery was as high as 41\% [8]. Minimisation of early failures, and the use of two new endoprosthetic systems, established total ankle replacement in a better position. Those new systems were Agility Total Ankle Replacement (TAR) and Scandinavian Total Ankle Replacement (STAR) [4]. The Agility prosthesis is most widely used in the United States. It is a semi-constrained, two-element, non-cement implant with a highly congruent talar element and polyethylene insert. The talar element has a porous cobalt-chromium coating, and the tibial element is coated with titanium. A polyethylene insert (fixed-bearing type) locked into the tibial element, together with the talar element, forms an articular surface. Since the massive tibial element covers articular surfaces of both malleoli, it is necessary to immobilise the tibiotalar syndesmosis. Movements within the non-immobilised syndesmosis lead to osteolysis of the distal end of the fibula, and require a revision arthrodesis of the ankle joint.

In a study by Knecht et al. on 126 patients with a mean follow-up of 9 years, the rate of revision surgery was $15 \%$. Pain relief and satisfaction were reported by 
$90 \%$ of patients. Complications included non-union of syndesmosis ( $8 \%)$, subsidence of one of the prosthetic elements (14\%), and radiological signs of osteolysis around the implant (76\%). A large proportion of patients developed degenerative lesions in the subtalar and talonavicular joints. Early and mid-term outcomes after Agility ankle replacement were good; however, the longterm survival rate at 11 years was only 60\%. Patients participating in the above-described study were selected by the author of the Agility system; all of them were ideal candidates for the surgery [9].

Spirit et al. described a group of 306 patients after Agility total ankle replacement, with a mean follow-up of 33 months. The five-year implant survival rate was $80 \%$. Nearly $54 \%$ of patients required revision surgery. Outcomes were definitely worse in younger patients of less than 55 years of age. Apart from typical postoperative complications, several patients required lower leg amputation as a lifesaving procedure. The authors of the study accepted less restrictive indications for the surgery, and the mean age of the patients was lower [7].

Another type of implant is the STAR system, which is most widely used in Europe. The prosthesis consists of tibial and talar elements, both made of coated alloys, and a mobile polyethylene insert. Scandinavian Total Ankle Replacement implants have a cylindrical shape, and show less rotational stress at the implant-bone interface. The tibial and talar elements are of similar sizes, and the polyethylene insert between them is mobile, which makes the prosthesis unconstrained. When implanted, the prosthesis does not exactly reproduce the articular surface, leaving part of the cartilage in the medial and lateral malleolar regions. The mobile insert is designed so that it can shift against the tibial and talar elements, and it absorbs compressive forces acting within the ankle joint. The mobile insert enhances structural freedom, which, however, increases the risk of prosthesis dislocation or luxation [4, 5].

Scandinavian Total Ankle Replacement ankle replacement outcomes have been analysed in numerous studies. A report describing 200 implants stated that 5 -year survival was $92.7 \%$. The most common complications include delayed wound healing, and lateral and medial malleolar fractures, with their incidence decreasing along with the rising surgeon's learning curve [10]. Other study results were less encouraging. In one study, the 5-year survival rate was only $70 \%$ [11]. In a prospective study of 200 prostheses, Wood et al. analysed midand long-term outcomes, and concluded that the 5-year and 10 -year survival rates were $93.3 \%$ and $80.3 \%$ respectively [10]. In 2004, Kofoed published study results of 55 patients who underwent ankle replacement with the use of cement or cementless STAR implants. The five- year survival rate was $90 \%$. The main cause of failures was aseptic loosening of the prosthesis. It was stressed that a major factor of arthroplasty success was proper selection of patients [12]. In materials published in 2013, Brunner et al. presented long-term outcomes of STARtype ankle replacement. Evaluation included 77 patients, mean age 56 years, with mean follow-up of 12.4 years. The prosthesis survival rate at 10 years was $70.7 \%$, and at 14 years $45.6 \%$. The main causes of arthroplasty failures were aseptic loosening of the prosthesis, subsidence of the talar element, formation of osteolytic cysts around the implant, and fracture of the polyethylene insert in 11 cases [13]. Pedersen et al. evaluated outcomes of ankle replacement in two groups of patients (100 patients). The first group included patients diagnosed with rheumatoid arthritis, and the other group included patients diagnosed with a degenerative disease. The mean follow-up was 63.8 months for the first group, and 65.9 for the second group. During the follow-up period, revision surgery was performed in 7 patients with rheumatoid arthritis, and 5 patients with a degenerative disease. Clinical outcomes were comparable for the two groups, and were assessed as good [14].

\section{Total ankle replacement - rehabilitation}

Patients qualified for total ankle replacement were those in whom joint injury caused limited mobility, pain, and gait pattern disorders. It is the only surgical procedure using an endoprosthesis where a plaster cast is applied immediately after surgery. It results from the specific anatomy of the joint, with stability of the joint largely depending on an efficient bone and ligament apparatus [15].

A plaster cast is applied after the postoperative wound is sutured, and usually remains in place for six weeks after the surgery. During that time, it is vital to have the operated leg elevated. Rehabilitation begins with breathing and antithrombotic exercises in a lying position. Then, the patient is seated with the legs hanging down for a few minutes. In the case of severe pain or a bursting feeling, the patient should return to a lying position. The patient's adaptation to the upright position takes place in the first week after surgery. Nonweight-bearing walking using crutches or a walker is allowed when the patient is able to keep the involved leg hanging down for at least 10-15 minutes. The plaster cast and the ban on weight bearing of the leg considerably delimit the physiotherapeutic techniques that may be used at the early stage of rehabilitation.

In the later applied plaster cast there is a hole that allows for physiotherapeutic procedures. At the initial stage, it is usually laser therapy, which has an analgesic and biostimulating effect [16]. In the case of extensive 
swelling of the operated limb, the full plaster cast may be replaced by an ankle-foot orthosis to enable cryotherapy or lymphatic drainage.

After the scheduled removal of the plaster cast, mobility of the joint is gradually restored. Initially, the range of motion is restored through active-passive exercises in the sagittal plane only, and within limits of pain tolerated by the patient. To make physical therapy more efficient, it is recommended to precede it with cryotherapy or lower leg massage. The treatments improve elasticity of lower leg muscles, and relieve the pain caused by calcaneal tendon (Achilles tendon) stretching. Where the involved joint is extremely stiff, a few days of traction therapy may be useful. Traction should be applied with little weight for 2 minutes at most. The procedure is repeated 2-3 times daily [3].

Active exercises may be initiated when the range of painless passive motion is achieved, sufficient for proper walking, that is, 20-degree flexion and 15-degree extension. Then, the gastrocnemius and soleus muscles are strengthened. First, the patient should perform active exercises in lying and sitting positions. As muscle strength increases, the position and difficulty of exercises are modified until the patient is able to stand on the toes in a standing position.

The dorsiflexion range should be increased through post-isomeric relaxation of flexors, and active exercises of extensors of the ankle joint.

Supination and pronation exercises may start only when complete active motions of flexion and extension are restored. Failure to follow that order may lead to destabilisation of the ankle joint and damage of the prosthesis fixing. As long as the patient feels pain with full weight-bearing of the involved leg, it is recommended to spare the leg by using crutches and orthopaedic footwear to stabilise the affected ankle joint [15].

It is estimated that gait function after total ankle replacement should be fully restored at one year after the surgery.

\section{Summary}

Total ankle replacement is a method of surgical treatment which seems to be an alternative to arthrodesis of the ankle joint in a selected group of patients. Progress in the design of endoprostheses has been made over the past few years. Their implantation technique has improved as well. Increased experience has led to a higher survival rate and lower complication rate as compared to first-generation prostheses. There are still unsolved problems concerning kinematics of the replaced joint. The range of motion following implantation remains limited to about 30 degrees. Gait studies indicate that even in the absence of complaints, weight-bearing of the joint remains limited, and its mobility changed. It has not been established yet whether total ankle replacement contributes to the development of degenerative lesions in adjacent joints. The ankle replacement procedure is subject to continuous modifications. The second generation of implants gained an advantage over previous cemented implants; however, no long-term prospective randomised studies have been conducted. Clinical outcomes of total ankle replacement are still worse than those achieved with other joints, such as hip and knee replacement $[4,5]$.

The authors declare no conflict of interest.

\section{References}

1. Błaszczyk JW, Czerwosz L. Stabilizacja posturalna w procesie starzenia. Gerontol Pol 2005; 13: 25-36.

2. Kapandji AJ. Anatomia funkcjonalna stawów. Gnat R (ed. Polish edition). Vol. 2. Urban \& Partner, Wrocław 2013.

3. http://www.fizjo-sport.pl/news/178/59/Anatomia-i-funkcjonalna-biomechanika-stawu-skokowego.

4. Yalamannchili P, Neufeld S, Lin S. Aloplastyka całkowita stawu skokowego - współczesna perspektywa. Górecki A (ed. Polish edition). Curr Orthop Pract 2009; 6.

5. Digovanni CW, Greisberg J. Foot and Ankle Core Knowledge in Orthopaedics. Marczyński W (ed.). Elsevier Urban \& Partner, Wrocław 2010

6. Haskell A, Mann RA. Perioperative complication rate of total ankle replacement is reduced by surgeon experience. Foot Ankle Int 2004; 25: 283-289.

7. Spirt AA, Assal M, Hansen ST Jr. Complication and failure after total ankle arthroplasty. J Bone Joint Surg Am 2004; 86-A: 1172-1178.

8. Valderrabano V, Hintermann B, Dick W. Scandinavian total ankle replacement: a 3.7-year average follow up of 65 patients. Clin Orthop 2004; 424: 47-56.

9. Knecht SI, Estin M, Callaghan JJ, et al. The Agility total ankle arthroplasty: Seven to sixteen year follow up. J Bone Joint Surg Am 2004; 86A-6: 1161-1171.

10. Wood PL, Deakin S. Total ankle replacement. The result in 200 ankles. J Bone Joint Surg 2003; 85(B): 334-341.

11. Anderson T, Montgomery F, Carlsson A. Uncemented STAR total ankle prostheses. J Bone Joint Surg 2004; 86(A) Suppl 1 (Pt2): 103-111.

12. Kofoed H. Scandinavian total ankle replacement (STAR). Clin Orthop Relat Res 2004; 424: 73-79.

13. Brunner S, Barg A, Knupp M, et al. The Scandinavian Total ankle replacement, Long term eleven to fifteen year Survivorship analysis of the prosthesis in seventy two consecutive patients. J Bone Joint Surg 2013; 95: 711-718.

14. Pedersen E, Pinsker E, Younger AS, et al. Outcome of total ankle arthroplasty in patients with rheumatoid arthritis and non-inflammatory arthritis: multicenter cohort study comparing clinical outcome and safety. J Bone Joint Surg Am 2014; 96: 1768-1775. 
15. Turski P. Fizjoterapia po operacjach synowektomii, endoprotezoplastyce i artroskopii. In: Fizjoterapia w reumatologii. Księżopolska-Orłowska K (ed.). Wydawnictwo Lekarskie PZWL, Warszawa 2013.

16. Kochański JW, Kochański M. Medycyna fizykalna. PHU Technomex, Gliwice 2009. 